\title{
Patología (pseudopatología) lingual observada en la facultad de odontología de la UCM
}

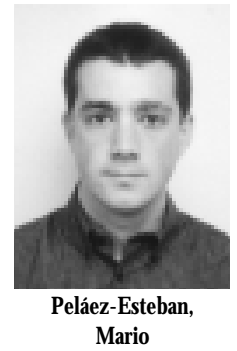

Lingual pathology (and pseudopathology) observed in the UCM Dental School (Madrid, Spain)

Peláez-Esteban, Mario*

Esparza-Gómez, Germán** Cancela-Rodríguez, Paloma*

Llamas-Martínez, Silvia***

*Licenciado en Odontología UCM

** Profesor Titular del Departamento de Medicina y Cirugía Bucofacial de la Facultad de Odontología de la UCM *** Profesora colaboradora honorífica del Departamento de Medicina y Cirugía Bucofacial de la Facultad de Odontología de la UCM.

\begin{abstract}
Resumen: Se ha realizado un estudio descriptivo, retrospectivo y transversal sobre la patología (pseudopatología) lingual observada en el Departamento de Medicina y Cirugía Bucofacial de la Facultad de Odontología de la UCM y se han comparado los resultados con otros estudios similares. De un total de 1313 historias clínicas revisadas, 54 (4,11\%) presentaron una o varias alteraciones linguales. La lengua geográfica fue la entidad más frecuentemente encontrada, en un $2,13 \%$ de los pacientes, seguida de la lengua fisurada, $1,07 \%$ y de la hipertrofia de las papilas foliadas con un $0,53 \%$ Las condiciones englobadas en el grupo de pseudopatología, son entidades muy próximas a la normalidad, con un diagnóstico que se solapa en ocasiones con lenguas sanas. El diagnóstico se produjo en un $27,8 \%$ de los casos de forma casual, no siendo el motivo de consulta del paciente al acudir al Departamento.
\end{abstract}

Palabras clave: Epidemiología oral, Patología lingual, Pseudopatología

Abstract: A descriptive, retrospective and cross-sectional study based on the lingual pathology (and pseudopathology) observed in the Department of Oral Medicine and Surgery, Dental School, Universidad Complutense, Madrid has been performed, and the results have been compared with other similar surveys. From 1313 clinical histories reviewed, 54 $(4,11 \%)$ presented one or more lingual alterations. The most frequently found condition was geographic tongue, $2,13 \%$ of the patients; followed by fissured tongue, $1,07 \%$ and foliate papilae hypertrophy $0,53 \%$

The lingual conditions included in the pseudopathology group are entities very close to normality, and the diagnosis frequently overlaps with healthy tongues. In $27,8 \%$ of the cases the diagnosis was fortuitous, with the lesion not being the cause of consultation.

Key words: Arthrocentesis, Oral epidemiology, Lingual pathology, Pseudopathology.

\begin{tabular}{ccc}
\hline Fecha recepción & Fecha última revisión & Fecha aceptación \\
$17-10-2003$ & $09-03-2004$ & $20-06-2004$ \\
\hline
\end{tabular}

Mario Peláez Esteban

Cl Cristóbal Bordiú 8 - 4ำ A

28003 - Madrid 


\section{Introducción}

Es notorio que existe una falta de datos en lo referente a la epidemiología de las lesiones que pueden afectar a la mucosa oral. Ya hace años se puso de manifiesto la preocupante falta de investigaciones epidemiológicas en las lesiones mucosas (con excepción quizá del cáncer y la leucoplasia). En opinión de algunos autores ${ }^{1}$, estas investigaciones se han iniciado muchos años más tarde que las referentes a la caries y la enfermedad periodontal (de las que sí se poseen datos más válidos) y cuando se ha realizado ha sido con poblaciones muy diferentes, sometidas a variados factores de riesgo y recogidas con distintos métodos, lo que conlleva que no se hayan podido obtener conclusiones generales ni a comparar los datos de unos estudios con otros. Concluían proponiendo la necesidad de llegar a un consenso de criterios diagnósticos en las distintas lesiones para estimar la prevalencia de las mismas y realizar estudios que permitan comparar poblaciones de distintas latitudes ${ }^{1}$. Más concretamente al referirse a la patología lingual, la escasez de estudios es aún más notable.

Por otro lado, la lengua presenta un tipo de patología muy específico. Ciertas alteraciones linguales suelen ser motivo de preocupación por parte de los pacientes, debido a que dicho órgano es fácilmente visible en la cavidad bucal, y puede variar su aspecto de forma considerable. Se trata de una serie de entidades clínicas que únicamente acontecen en el órgano lingual, presentan una serie de características comunes, y merecen ser estudiadas en conjunto. Dichas entidades son consideradas por algunos autores como patológicas, mientras que otros opinan que son variaciones de la normalidad sin significación clínica (pseudopatológicas) ${ }^{2 * *}$. Estas condiciones linguales presentan etiología desconocida, clínica florida y diagnóstico sencillo.

La palabra pseudopatología es un término acuñado en 1994 por Cawson $^{2 *}$, y se refiere al conjunto de dichas entidades. Su importancia radica principalmente en saber diagnosticarlas, conociendo sus factores predisponentes, y en realizar un diagnóstico diferencial con otros procesos similares. Sin embargo, en ocasiones pueden presentar sintomatología asociada, o requerimientos terapéuticos que pueden hacer pensar que se trate de procesos realmente patológicos.

Por otro lado, creemos que sería interesante estudiar la patología lingual observada en un Departamento de Medicina Oral, y compararla con otros estudios similares.

Los objetivos que se pretenden conseguir con este trabajo son: en primer lugar, revisar las historias clínicas de Medicina Bucal del Departamento de Medicina y Cirugía Bucofacial de la Facultad de Odontología de la UCM y seleccionar aquellas en las que fueron diagnosticadas una 0 varias alteraciones linguales. No han sido consideradas en este estudio aquellas alteraciones o lesiones que pueden acontecer en cualquier localización de la mucosa oral sin ser propias de la lengua, como por ejemplo el liquen plano, la leucoplasia o el pénfigo vulgar. Posteriormente se realizó un estudio epidemiológico con los datos obtenidos de dichas historias, y finalmente se compararon los resultados con los de estudios similares referidos en la literatura.

\section{Material y Método}

Se ha realizado un estudio descriptivo, observacional, retrospectivo y transversal empleando la base de datos de Medicina Bucal del Departamento de Medicina y Cirugía Bucofacial de la Facultad de Odontología de la Universidad Complutense de Madrid, revisando las historias clínicas de los pacientes que acudieron para la consulta de alguna afección o lesión en la cavidad oral entre el 1 de marzo de 1982 y el 30 de enero de 2003. Se seleccionaron las historias que presentaban algún tipo de patología propiamente lingual, es decir, aquella patología que sólo se puede presentar en la lengua. De los 1313 pacientes que acudieron a este Departamento, en 54 se detectó algún tipo de alteración lingual, ya fuera como motivo de consulta, o en forma de hallazgo casual durante la exploración.

\section{Resultados}

El total de la muestra estuvo constituido por 1313 pacientes, que acudieron al Departamento de Medicina y Cirugía Bucofacial de la Facultad de Odontología de la UCM entre el 1 de marzo de 1982 y el 30 de enero de 2003. 54 de éstos presentaron algún tipo de alteración lingual $(4,11 \%)$, siendo 24 varones (43,4\%de los mismos) y 30 mujeres (55,6\%). El rango de edad de estos 54 pacientes se situó entre los 4 y los 78 años, hallándose una media de 46,9 años (fig. 1).

Respecto a la remisión de los pacientes al Departamento, el 64,8\% acudieron remitidos por un dentista privado, el $18,5 \%$ fueron derivados des-

RCOE, 2004, Vol 9, №4, 381-390 


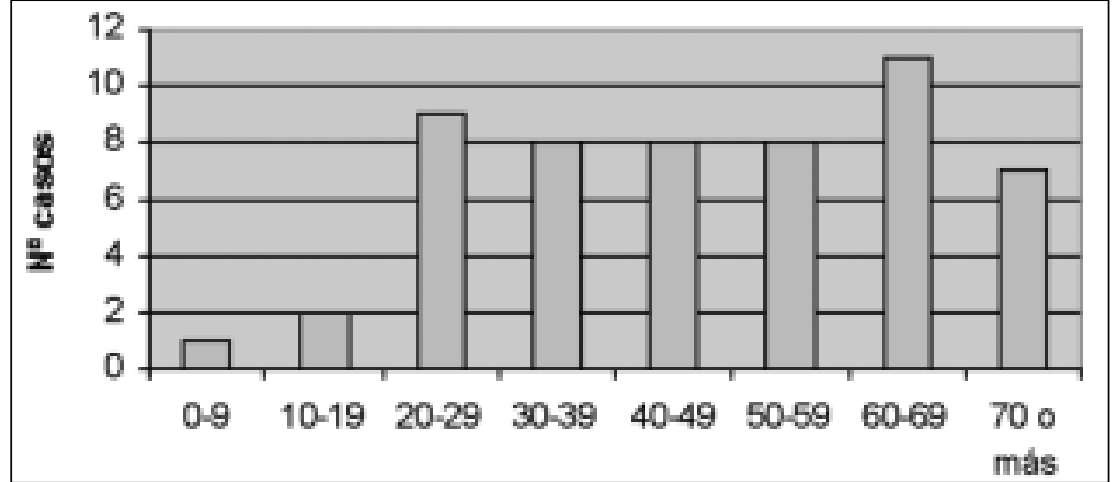

Figura 1. Distribución por grupos de edad.

de la propia Facultad, bien desde la recepción general o bien desde los diferentes postgrados, y el 5,6\% por su médico general de la Seguridad Social. La tabla 1 refleja estos datos de forma detallada.

Entre los pacientes con algún tipo de patología lingual, se encontraron los diagnósticos que aparecen detalla- dos en la figura 2 . El $4,11 \%$ de los pacientes que acudieron al Departamento, fue diagnosticado de alguna alteración lingual. La lengua geográfica fue la entidad que más se repitió entre los pacientes que acudieron al Departamento, estando presente en el 2,13\% (28 pacientes). La lengua fisurada fue la segunda característica

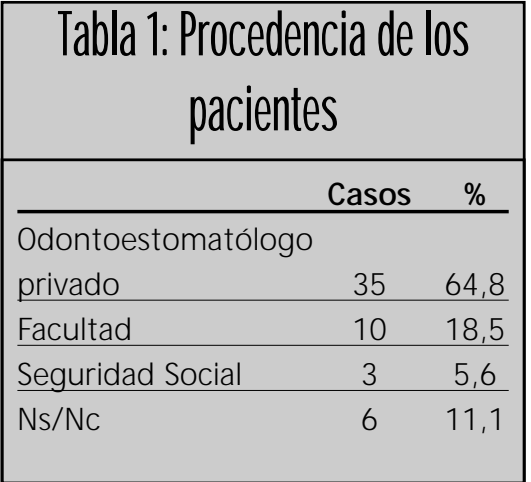

más comúnmente observada, y se encontró en el 1,07\% En tercer lugar, apareció la hipertrofia de las papilas foliadas con un $0,53 \%$ de prevalencia. En total, en los 54 pacientes, se diagnosticaron 74 entidades; es decir, cada paciente presentó una media de 1,4 alteraciones linguales. 34 de los pacientes (63\%) presentaron un único

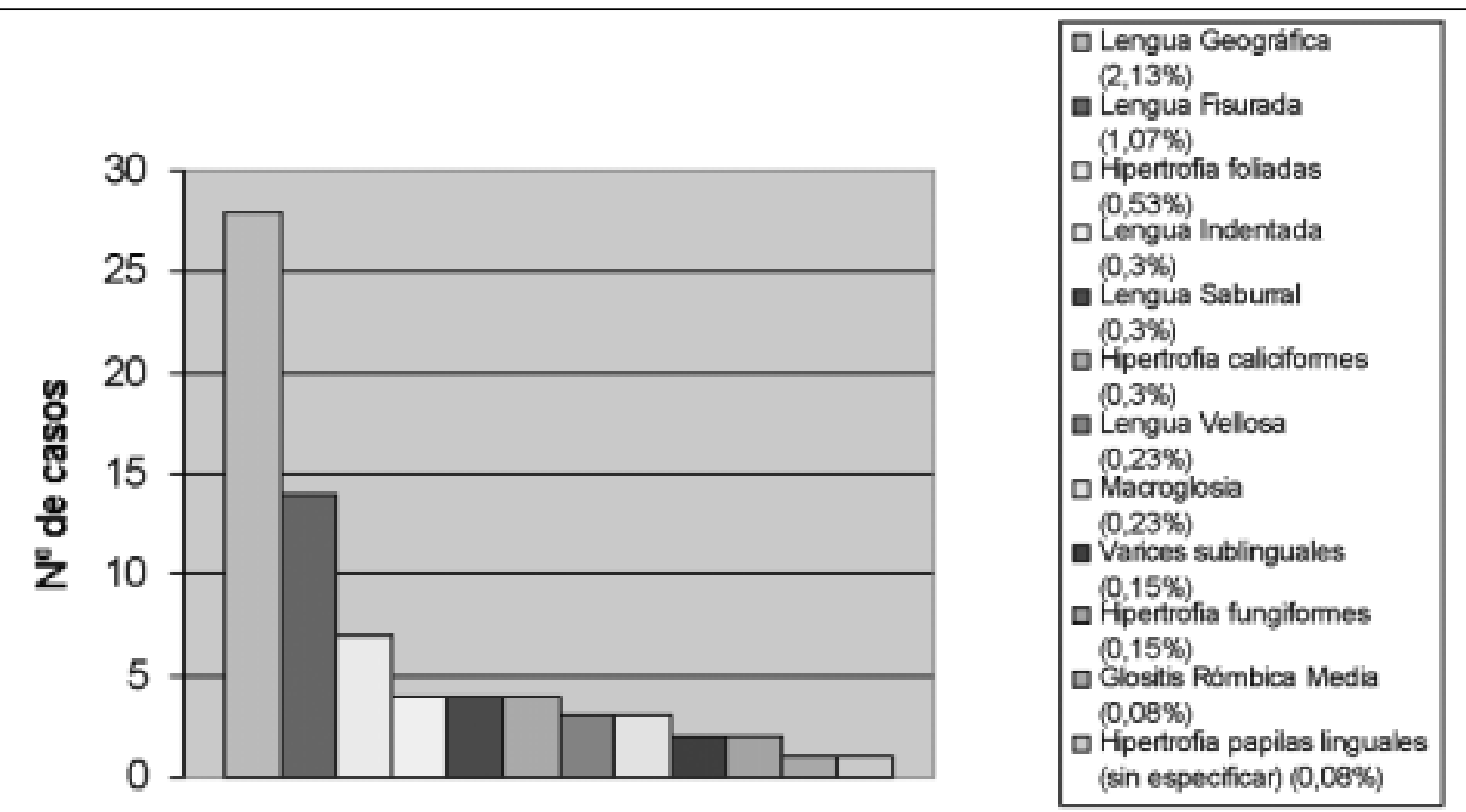

Figura 2. Entidades diagnosticadas 


\begin{tabular}{|lcc|}
\hline \multicolumn{3}{|c|}{ Tabla 2: Sintomatología } \\
asociada \\
\hline Síntomas & Casos & $\%$ \\
\hline Ninguno & 27 & 50 \\
Escozor & 11 & 20,4 \\
Molestias & 9 & 16,7 \\
Quemazón & 5 & 9,3 \\
Dolor & 4 & 7,4 \\
Irritación & 3 & 5,6 \\
Ardor & 1 & 1,9 \\
Hinchazón & 1 & 1,9 \\
Boca seca & 1 & 1,9 \\
\hline
\end{tabular}

diagnóstico lingual; por tanto, el resto tuvo una media de dos entidades diagnosticadas.

Los pacientes fueron también preguntados por los síntomas asociados a su afección lingual, obteniéndose los resultados que se presentan en la tabla 2. Los síntomas aparecen reflejados tal y como los pacientes los describieron. El dato más significativo resultó ser que el $50 \%$ de los pacientes no describió ningún síntoma. Los pacientes que

\begin{tabular}{|c|c|c|}
\hline \multicolumn{3}{|c|}{$\begin{array}{l}\text { Tabla 3: Causas a las que los } \\
\text { pacientes atribuyeron la } \\
\text { existencia de las alteraciones } \\
\text { linguales }\end{array}$} \\
\hline & Casos & $\%$ \\
\hline $\begin{array}{l}\text { Extracción terce } \\
\text { molares }\end{array}$ & 5 & 9,3 \\
\hline Alergias & 5 & 9,3 \\
\hline Prótesis dental & 4 & 7,4 \\
\hline $\begin{array}{l}\text { Alteraciones } \\
\text { emocionales }\end{array}$ & 3 & 5,6 \\
\hline $\begin{array}{l}\text { Sin relación con } \\
\text { algún factor }\end{array}$ & 2 & 3,7 \\
\hline $\mathrm{Ns} / \mathrm{Nc}$ & 35 & 64,8 \\
\hline
\end{tabular}

presentaron síntomas emplearon para describirlos una media de 1,3 términos. Entre los síntomas expresados por los pacientes, un $20,4 \%$ refirió escozor, un $16,7 \%$ molestias, un $9,3 \%$ quemazón y un $7,4 \%$ dolor.

Se les preguntó si atribuían su afección lingual a algún suceso o causa. Las respuestas quedan reflejadas

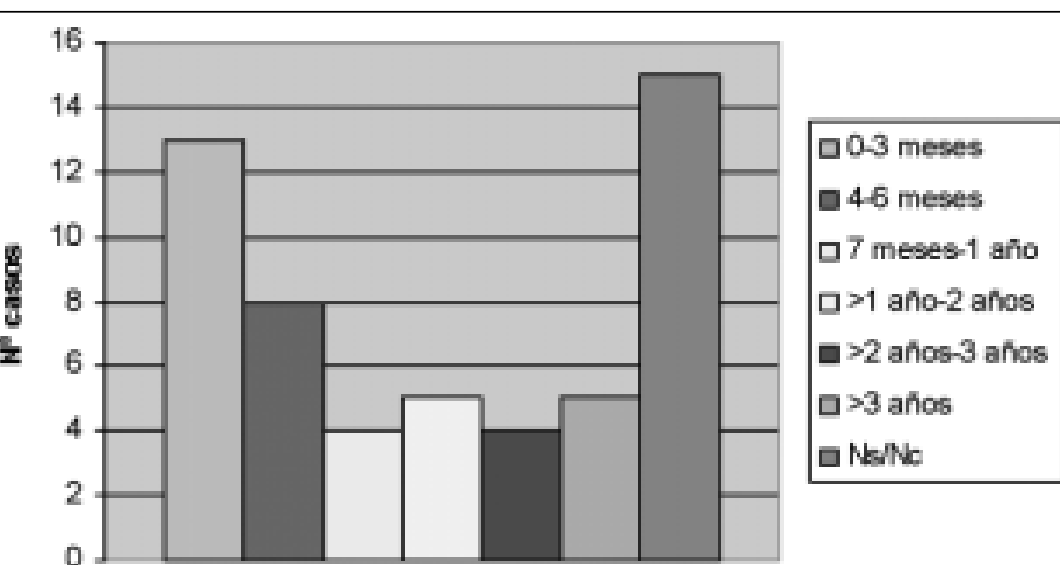

Figura 3. Tiempo de evolución.

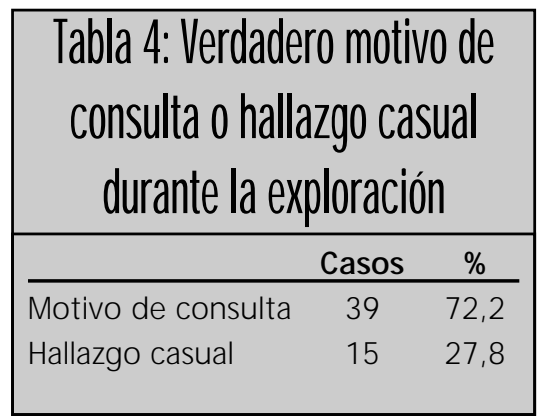

en la tabla 3. En primer lugar, un $9,3 \%$ de los pacientes lo atribuyeron a la extracción de un tercer molar, y en el mismo porcentaje lo relacionaron con una alergia. En el 7,4\% lo atribuyeron a una prótesis dental y el 3,7\% no lo relacionó con ningún factor. En relación con los antecedentes familiares, el $96,3 \%$ de los pacientes no refirió ninguno.

Se investigó asimismo si los pacientes acudieron con un motivo de consulta en relación con alguna de las alteraciones linguales estudiadas o si dichas entidades fueron hallazgos diagnósticos casuales durante la exploración de otra patología. En la tabla 4 observamos que en un $27,8 \%$ de los casos, las condiciones linguales descritas fueron un hallazgo casual.

En referencia al tiempo de evolución que llevaba presente la entidad lingual, de los 54 pacientes, en 15 historias no se pudo determinar. Entre los 39 que sí lo refirieron, se observó un rango entre un día y 18 años. Al hacer los cálculos estadísticos, se obtuvo una distribución trimodal (6, 12 y 36 meses de evolución), con una media de 22,9 meses y una mediana de seis meses (fig. 3).

Se observó que una parte de los pacientes presentaban asociada algu- 


\begin{tabular}{|c|c|c|}
\hline \multicolumn{3}{|c|}{$\begin{array}{l}\text { Tabla 5: Pruebas diagnósticas } \\
\text { complementarias solicitadas }\end{array}$} \\
\hline Pruebas & Casos & $\%$ \\
\hline Biopsia & 2 & 3,7 \\
\hline Otras & 1 & 1,9 \\
\hline $\begin{array}{l}\text { Ninguna prueba } \\
\text { solicitada }\end{array}$ & 51 & 94,4 \\
\hline
\end{tabular}

\begin{tabular}{|lcc|}
\hline \multicolumn{3}{|l|}{ Tabla 6: Tratamientos aplicados } \\
\hline Tratamiento & Casos & $\%$ \\
\hline Ninguno & 47 & 87 \\
\hline Resina de podofilino & 3 & 5,6 \\
\hline Antifúngicos & 2 & 3,7 \\
\hline Otros & 2 & 3,7 \\
\hline
\end{tabular}

na alteración de tipo nervioso, por lo que se consideró oportuno incluir este apartado, ya que en algunas de estas entidades, el estrés o las alteraciones emocionales han sido descritas como posibles causas. El 33,3\% de los pacientes presentaron algún tipo de trastorno, que variaba desde un estado de estrés o cancerofobia a consecuencia de su alteración lingual, hasta cuadros depresivos en tratamiento.

En relación a las pruebas complementarias solicitadas, sólo se realizaron dos biopsias y un cultivo de hongos (tabla 5). Se evaluaron asimismo los tratamientos prescritos a aquellos pacientes que presentaron algún tipo de afección lingual, obteniéndose los resultados reflejados en la tabla 6 . En el $87 \%$ de los pacientes no se consideró necesaria la aplicación de ningún tratamiento. La resina de podofilino en solución acuosa, empleada para el tratamiento de los tres casos $(5,6 \%)$ de

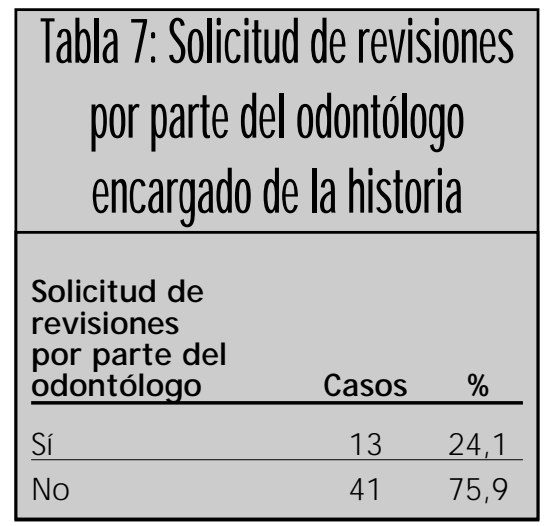

lengua vellosa, obteniéndose su curación en los tres, y la aplicación de antifúngicos en dos casos $(3,7 \%)$ fueron los dos tratamientos más empleados.

Se analizó también si el clínico recomendó al paciente acudir a revisiones periódicas, para evaluar la importancia que éste le daba a la alteración. En el $75,9 \%$ de los casos se consideró que no era necesario realizar revisión de las lesiones. Del mismo modo, se evaluó si los pacientes acudieron a las revisiones solicitadas, para valorar la trascendencia que le daban a su problema. Los resultados aparecen reflejados en las tablas 7 y 8 .

\section{Discusión}

De los 54 pacientes estudiados, el $55,6 \%$ fueron mujeres. Existe pues una leve mayoría de pacientes del sexo femenino, no significativa.

Casi dos tercios de los pacientes acudieron remitidos por su dentista $(64,8 \%$, aproximadamente una quinta parte desde la Facultad (18,5\%), mientras que en un bajo porcentaje $(5,6 \%)$ lo hicieron a través de su médico de cabecera en la Seguridad Social.

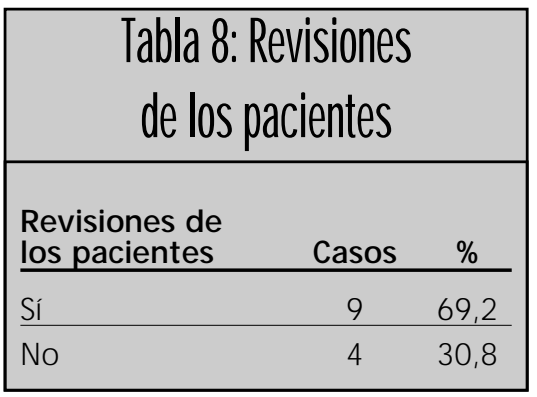

Parece desprenderse que el paciente suele realizar la consulta sobre su afección lingual a su dentista más que a su médico de cabecera, por lo que espera que éste le resuelva su problema. Por los datos obtenidos, esto no parece ser así, ya que los pacientes son remitidos al Departamento de la Facultad. El clínico generalista debería estar perfectamente capacitado para diagnosticar este tipo de afecciones, sin necesidad de remitirlas, ya que su diagnóstico clínico es sencillo, por lo que debería ser él quien tranquilizase al paciente.

En relación con los diagnósticos que se establecieron, cabe destacar que en ocho casos se presentó la lengua fisurada asociada con una lengua geográfica, es decir, en un $14,8 \%$ de los pacientes estudiados apareció esta asociación, a la que algunos denominan síndrome de lengua fisurada $a^{3,4}$.

En la literatura revisada, hemos encontrado trabajos de estudios epidemiológicos de dos tipos: unos referentes a patología oral en general, y otros específicamente linguales. Estos últimos, los más interesantes para este estudio, existen en menor cantidad. Por otro lado, la mayoría de ellos no trata este tipo de cuadros en concreto, sino que se refieren a cualquier patología que pueda aparecer en la 
lengua, como el liquen plano, úlceras, o papilomas, por poner algunos ejemplos, sin ser patología propia de la lengua. Del mismo modo, debemos reseñar que algunos de los trabajos presentaban prevalencias de estos cuadros en porcentajes únicamente sobre los pacientes con patología. Para poder comparar estos trabajos con los demás, así como con nuestros resultados, hemos tenido que calcular los porcentajes de dichas entidades sobre el total de pacientes revisados en cada estudio. Es necesario señalar asimismo que este estudio, al estar basado en los pacientes que acudieron a la Facultad de Odontología de la UCM, tiene un sesgo implícito, ya que no maneja la población general, sino que se centra en individuos que ya tienen alguna patología.

En primer lugar observaremos los datos recogidos en los estudios exclusivamente de patología lingual. En un trabajo de Richardson con 3319 estudiantes de raza negra ${ }^{5}$, se obtuvo una prevalencia de lengua geográfica del $1,08 \%$ y de la glositis rómbica media del 0,15\% Sobre 1017 niños húngaros de entre 1 y 14 años, Vörös-Balog y cols $^{6}$ hallaron que el $35,11 \%$ presentaba alguna lesión lingual, un porcentaje mucho mayor que el reflejado en nuestro estudio (4,11\%). Además, resulta interesante destacar que es uno de los pocos trabajos en los que la lengua fisurada $(29,2 \%)$ aparece en un porcentaje mayor que la lengua geográfica $(5,7 \%)$ en edades infantiles, ya que como veremos más adelante, la mayor parte de los estudios reflejan lo contrario. Redman ${ }^{7 *}$ evaluó la prevalencia de varias alteraciones linguales en 3611 niños. La entidad más frecuente resultó ser, al igual que en nuestro trabajo, la lengua geográfica, con un $1,41 \%$ seguida, también como en nuestro estudio, de la lengua fisurada con un $1,08 \%$ La glositis rómbica media se presentaba en un $0,14 \%$ de los niños, mientras que la lengua vellosa acaecía en un 0,06\% El estudio se basa sólo en niños, por lo que no se puede comparar con nuestros resultados, principalmente por la escasa incidencia de la lengua vellosa, ya que es una afección que se presenta preferentemente en adultos. Por otro lado, este es el único trabajo encontrado en el que aparece reflejada la glositis rómbica en niños con una incidencia considerable y uno de los argumentos por el cual no se considera una alteración del desarrollo es que no suele aparecer en niños. Este dato estaría, pues, en contra de dicha aseveración, aunque habría que reevaluar los criterios diagnósticos que existían en 1970 para determinar que la alteración era una glositis rómbica media.

Kullaa-Mikkonen ${ }^{8}$ realizó un estudio epidemiológico en Finlandia, en el que halló que la principal alteración lingual era la lengua vellosa, con un $8,4 \%$ No concuerda con los demás trabajos ni con nuestros resultados, posiblemente porque incluye la lengua saburral en el diagnóstico, por lo que los datos no pueden ser comparables. En el mismo estudio, la lengua fisurada era la segunda alteración lingual más frecuente, con un $5 \%$ mientras que la lengua geográfica estaba presente en un 2,1\%

En un estudio de screening de lesiones linguales en una población húngara ${ }^{9}$, sobre un total de 7820 individuos, se hallaron alteraciones linguales en el 18,52\% por tan sólo un 4,11\% en nuestro estudio, siendo la más fre- cuente la lengua fisurada con un 8,8\% seguida de la lengua geográfica con un $3 \%$ y la glositis rómbica media con un $0,35 \%$ El resto de lesiones observadas escapan al objetivo de este trabajo, por lo que no se comentan.

En otro estudio en 1013 pacientes jordanos que acudieron a la consulta dental ${ }^{10}$, se estudió la incidencia de lesiones linguales. El 23,7\% presentaban algún tipo de alteración lingual, lo que es un resultado muy alto, ya que prácticamente uno de cada cuatro pacientes fueron diagnosticados de alguna alteración lingual. En nuestro estudio tan sólo el 4,11\%de los pacientes presentaron alguno de los cuadros objeto de este trabajo. La lengua fisurada fue la más comúnmente diagnosticada, con un $11,4 \%$ seguida de la lengua geográfica con el $6,8 \%$ la lengua vellosa con el 3,4\% con una clara predisposición por el sexo masculino, y la glositis rómbica media con el $0,4 \%$

En segundo lugar veremos algunos estudios epidemiológicos orales, no simplemente linguales. Sawyer ${ }^{11}$, sobre 2203 niños nigerianos, observó lengua fisurada en el $0,8 \%$ de los casos, lengua geográfica en el 0,3\% y anquiloglosia en el 0,2\% Salem ${ }^{12}$ encontró en una muestra de 1932 niños para un estudio de lesiones orales, el 0,8\% de lengua fisurada, $0,2 \%$ de lengua geográfica y $0,1 \%$ de anquiloglosia. Cabe destacar que no se observó ninguna glositis rómbica media en ninguno de los dos estudios ${ }^{11,12}$, ya que como hemos dicho anteriormente, sólo encontramos un autor que describió dicha entidad en niños con una incidencia significativa. Los porcentajes de estos estudios son menores comparados con los de nuestro traba- 
jo, así como con aquellos realizados en estudios específicamente linguales. Esto puede deberse, en nuestra opinión, a una falta de interés hacia este tipo de patología, ya que en un estudio oral pueden ser diagnosticadas entidades de peor pronóstico, en las cuales se centra posiblemente el clínico a la hora de la exploración.

En un trabajo de patología oral ${ }^{4}$, sobre 1609 pacientes eslovenos, se encontró un $21,1 \%$ de individuos con lengua fisurada, un $16,2 \%$ con varices sublinguales y un 2,2\% con lengua geográfica. Todas estas incidencias parecen anormalmente elevadas, posiblemente debido a que el diagnóstico de estas alteraciones utiliza criterios diferentes.

En un estudio de Sedano y cols $^{13}$ de alteraciones orales en México, sobre una muestra de 32022 niños, se hallaron las siguientes incidencias: lengua fisurada un $15,7 \%$ y lengua geográfica un $1,9 \%$

Guggenheimer y cols ${ }^{14}$ observaron en un estudio en pacientes diabéticos los siguientes resultados: en el grupo control, la incidencia de la lengua fisurada fue del $0,4 \%$ mientras que en el grupo de diabéticos, aumentaba hasta un 5,4\% la glositis rómbica y la lengua geográfica también fueron netamente más frecuentes en el grupo diabético $(7,2 \%$ y $5,4 \%$ respectivamente), que en el grupo control $(0,4 \%$ y $3,4 \%$.

Kleinman y cols $^{15^{*}}$, sobre 39206 niños, vieron que la lengua geográfica resultó ser la cuarta lesión oral más frecuente, representando además el $76,8 \%$ de las alteraciones de la lengua. En otro estudio en niños en Sudáfrica ${ }^{16}$, se observó lengua geográfica en el 1,6\%de los individuos (siendo la cuarta lesión oral más prevalente) y lengua fisurada en el 0,6\% Parece ser que en los estudios en niños ${ }^{15^{*}, 16,17}$, efectivamente la lengua geográfica suele aparecer en mayor proporción que la lengua fisurada, mientras que en estudios en adultos ${ }^{4,8,10,18-20}$, ocurre al revés. Esto podría estar en concordancia con la teoría de que ambas entidades forman parte de una sola que va evolucionando con el paso del tiempo, el síndrome de lengua fisurada.

En un trabajo en sujetos de más de 40 años $^{21}$, se halló que la lesión oral más frecuente era la lengua saburral, en el $47,45 \%$ de los pacientes. No se trata del único trabajo revisado en el que aparece como la lesión más diagnosticada, ya que en un estudio de García-Pola y cols ${ }^{17}$ en Oviedo, sobre niños de 6 años, también fue la entidad más frecuente con un $16,02 \%$

Ikeda y cols $^{22}$, en una muestra de 1319 individuos camboyanos, no hallaron ninguna de las alteraciones estudiadas en este estudio. Esto puede deberse, como ya hemos comentado, a una falta de atención del clínico hacia esta patología o pseudopatología, ya que posiblemente varios de los individuos examinados presentaban algún cuadro lingual, pero quizá no fue trascendente clínicamente para el examinador, o lo pasó por alto.

Zain y cols $^{23}$, sobre 11707 pacientes, vieron que el 0,33\% presentaba una glositis rómbica media, y ninguna otra lesión lingual fue evaluada. En un screening realizado en soldados, sobre una muestra de 181338 individuos, la lengua geográfica y la glositis rómbica media, ambas con 12 casos (un $0,007 \%$ y la lengua vellosa negra $(0,003 \%)$ fueron las únicas lesiones linguales que se diagnosticaron ${ }^{24}$. Estas incidencias son anormalmente bajas comparadas con nuestros resultados, así como en relación con otros estudios revisados. De nuevo debemos observar que quizá se deba a que el examinador no se percatara de la presencia de estas alteraciones, la considerara una variación de la normalidad, o simplemente no le diera importancia.

En otro estudio de screening sobre 23616 individuos blancos norteamericanos $^{25}$, cinco de los cuadros estudiados en este trabajo aparecen entre las 25 lesiones orales más frecuentemente observadas: la lengua fisurada $(0,34 \%)$, las varicosidades sublinguales $(0,32 \%)$, la lengua geográfica $(0,31 \%)$, la lengua vellosa negra $(0,11 \%)$ y la glositis rómbica media $(0,09 \%)$. Estos resultados se asemejan bastante a los hallados en nuestro trabajo, ya que tan sólo la lengua geográfica $(2,13 \%)$ presenta una prevalencia alejada de nuestros datos.

En un estudio de García-Pola y Martínez-Díaz ${ }^{2 * *}$ en la Universidad de Oviedo, se evaluó la prevalencia de la patología oral en pacientes de la Escuela de Estomatología de Oviedo. Este trabajo se basa, al igual que el nuestro, en los pacientes que acudieron a un centro docente y asistencial. En este tipo de pacientes, el porcentaje de patología oral es considerablemente mayor que en los que pertenecen a estudios de población general. En este estudio ${ }^{26^{* *}}$, la patología lingual más frecuente fue la presencia de varices linguales, con un $13,6 \%$ Las otras dos únicas lesiones linguales diagnosticadas fueron la lengua fisurada $(5,6 \%$ y la lengua vellosa $(1,6 \%$. Estos datos son más elevados que los hallados en nuestro estudio. 
Es interesante destacar que en los estudios orales, las prevalencias de estas entidades descienden considerablemente, posiblemente a causa de que no resultan relevantes para el clínico que examina a los pacientes, ya que otras entidades, netamente patológicas, resultan más interesantes. Esto apoya la teoría de que mucha de esta patología o pseudopatología lingual no tiene trascendencia clínica.

Con un análisis global de estos datos epidemiológicos podemos observar que las diferencias entre los distintos trabajos son abismales en algunos casos. Esto puede deberse, en nuestra opinión, a que estamos ante un tipo de cuadros que en muchas ocasiones no generan sintomatología y que están próximos a la normalidad anatómica. Por ello, en ocasiones se pasa por alto su diagnóstico, mientras que en otras se toma por una entidad de las estudiadas en este trabajo una lengua normal.

Debemos destacar que el $50 \%$ de los pacientes no describieron ningún síntoma. Sin embargo, podemos considerar que existen además otros casos de pacientes que pueden presentar alguno de estos cuadros, pero que sin embargo no acuden a su dentista para consultarle debido a que no les genera ningún tipo de trastorno. En ese caso, este porcentaje teórico puede ser en la práctica mucho mayor. Cabe destacar que ciertos síntomas son asimilables entre sí: ardor, escozor o quemazón parecen referirse a un mismo efecto, aunque como se ha dicho, aparecen reflejados tal y como fueron descritos por los pacientes (tabla 2). La sintomatología más referida por los pacientes fue una sensación de escozor o quemazón, así como molestias.
En cuanto a la causa con la que relacionaron su afección, tan sólo un 3,7\% no lo relacionó con nada, mientras que las causas odontológicas, alérgicas o emocionales, fueron las más comúnmente aducidas. Resulta muy característico que a pesar de tener un tiempo de evolución largo, los pacientes lo relacionaron con facilidad con alguna intervención odontológica. Al investigar con detalle dicha intervención, ésta ocurrió generalmente varios meses antes de que el paciente notase algún signo o síntoma.

Tan sólo dos pacientes (3,7\%) refirieron tener antecedentes familiares de afecciones linguales. Este bajo porcentaje se puede explicar por varios motivos: en primer lugar, muchos de los pacientes ni siquiera eran conscientes de la presencia de alguna alteración lingual. En segundo lugar, entre aquellos que lo eran, un gran porcentaje no le daba importancia, por lo que tampoco se percataría de la presencia de los mismos signos en otros familiares. En tercer lugar, resulta poco valorable la asociación familiar en este tipo de entidades, aunque algunas tengan una posible influencia hereditaria como la lengua fisurada o la lengua geográfica.

Consideramos asimismo interesante estudiar si los pacientes acudieron con un motivo de consulta relacionado con las entidades linguales posteriormente diagnosticadas o por el contrario fueron hallazgos casuales durante la exploración de otra patología. Entre aquellos casos en los que la afección lingual fue el verdadero motivo de consulta, una gran parte fueron pacientes a los que sus dentistas remitieron al Departamento al observar de forma casual durante una exploración rutinaria la afección lingual, como hemos visto anteriormente. Por lo tanto, pese a que el dato teórico es que un $72,28 \%$ de los pacientes acudieron con ese motivo de consulta, en la práctica este porcentaje debe ser menor. Así pues, se observa que una buena proporción pertenece a aquellos que desconocían la presencia de cualquier alteración lingual, a los que habría que sumar los pacientes que también la desconocían cuando acudieron a su dentista, a su médico de cabecera o a aquella persona que les remitió al Departamento.

De los 54 pacientes, en 15 historias no se pudo determinar el tiempo de evolución. Esto puede deberse a que muchos no eran conscientes siquiera de su afección, y en otros casos no se preguntó, ya que el verdadero problema del paciente era otro. Debido al amplio rango en el que los pacientes cifraron el tiempo de evolución (entre un día y 18 años), parece más lógica la orientación por la mediana que por la media, por lo que se observa que la mayoría de los pacientes presentan una evolución de seis meses. De esta forma, se trata de cuadros a los que el paciente no da la importancia necesaria como para acudir con urgencia a una consulta dental, ya que los dejan evolucionar durante varios meses. Del mismo modo, podemos pensar que es el odontoestomatólogo privado el que decide remitir al paciente, advirtiéndole del cuadro lingual que presenta, al Departamento de la Facultad. Hasta ese momento, transcurridos varios meses desde su inicio, el paciente posiblemente no se había preocupado por su afección.

Un tercio de los pacientes refirieron algún tipo de trastorno emocio- 
nal, que variaba desde el estrés hasta la depresión, por lo que podemos considerar que pudiera ser un factor causal o favorecedor para la aparición de alguno de estos cuadros.

Observamos que en la mayoría de los casos $(94,4 \%)$ no se solicitó una biopsia ni ninguna otra prueba diagnóstica (tabla 5). Esto demuestra que el diagnóstico de este tipo de cuadros linguales es fundamentalmente clínico, sin necesidad de pruebas de otro tipo. En tan sólo dos casos se solicitó una biopsia debido a una alteración lingual entre aquellas incluidas en este estudio. En ambos casos se trataba de una lengua geográfica (una de ellas asociada a una lengua fisurada). Cabe destacar asimismo que en un estudio histológico de Weir y cols $^{27}$, sobre 15783 biopsias de lesiones orales, ninguna de ellas correspondía a alguna de las alteraciones linguales revisadas en este trabajo. Esto resalta no sólo la benignidad de estas lesiones, sino también el diagnóstico sencillo que presentan (ya que ninguna fue biopsiada para descartar otra patología más severa).

En la mayor parte de los pacientes (87\%), no fue necesario aplicar ningún tratamiento. En esos casos, lo único que se debía hacer era tranquilizar al paciente, explicando el alcance del problema. Parece pues evidente que estos cuadros no requieren tratamiento en su mayoría. La única entidad en la que se suele aplicar algún tratamiento protocolizado es la lengua vellosa.
En cuanto a las revisiones, en más de las tres cuartas partes de los casos, el dentista encargado de la historia no solicitó al paciente acudir a revisiones, al considerar que el cuadro no representaba ningún riesgo (tabla 7); una vez explicado el alcance del problema, no son necesarias las revisiones. A los que se recomendó volver al Departamento, algo más de un 30\% faltó a la revisión (tabla 8). Esto parece dejar claro que una vez explicado el problema al paciente, éste ya no le da importancia. Así, tanto el dentista como el paciente son conscientes de la escasa relevancia clínica de estos cuadros linguales.

\section{Conclusiones}

1. A la vista de la revisión bibliográfica realizada, cabe considerar que la lengua geográfica, la lengua fisura$\mathrm{da}$, la lengua vellosa, la lengua saburral, la glositis rómbica media, la lengua dentada, las varices sublinguales y las hipertrofias de las papilas foliadas, fungiformes y circunvaladas pueden ser consideradas como pseudopatología lingual, puesto que suelen ser asintomáticas, y no requieren la instauración de un tratamiento.

2. Las condiciones englobadas en el grupo de pseudopatología, son entidades muy próximas a la normalidad, con un diagnóstico que se solapa en ocasiones con lenguas sanas.
3. En el estudio realizado entre los pacientes que acudieron a la consulta de Medicina Bucal del Departamento de Medicina y Cirugía Bucofacial de la Facultad de Odontología de la UCM, las lesiones y pseudolesiones más prevalentes has sido las lengua geográfica, lengua fisurada e hipertrofia de las papilas foliadas, con una prevalencia global de $4,11 \%$ La mitad de estos pacientes no refirió ningún síntoma consecuente con su alteración lingual.

4. El diagnóstico se produjo en un $27,8 \%$ de los casos de forma casual, no siendo el motivo de consulta del paciente al acudir al Departamento. Este dato es en la práctica mucho mayor, ya que una parte de los pacientes que presentaban alguna de las entidades linguales revisadas en este trabajo fueron remitidos por su odontoestomatólogo privado, en ocasiones a raíz de un diagnóstico casual en su consulta.

5. Sería aconsejable, de cara a realizar bases de datos más fiables, que se anotaran como segundo o tercer diagnóstico las alteraciones linguales presentes para poder establecer su prevalencia real, ya que es razonable pensar que en muchas ocasiones se señala únicamente el diagnóstico principal, y no otras entidades linguales como las que se han revisado en este trabajo.

Pese a todo, se deben manejar todos estos datos con cautela ya que se trata de un pequeño número de casos, por lo que estas conclusiones deben ser observadas con reservas. 


\section{Bibliografía recomendada}

Para profundizar en la lectura de este tema, el/los autor/es considera/an interesantes los artículos que aparecen señalados del siguiente modo: *de interés $* *$ de especial interés.

1. Kleinman DV, Swango PA, Mindborg JJ, Gupta P. Toward assesing trends in oral mucosal lesions: lessons learned from oral cancer. Adv Dent Res 1993;7:32-41.

2**. Cawson RA, Everson JW. Oral pathology and diagnosis: a colour atlas and integrated text. Londres: Gower, 1994:1.12-1.16.

Es el primer autor que empleó el término de pseudopatología lingual.

3. Kullaa-Mikkonen A, Penttila I, Kotilainen R, Puhakainen E. Haematological and immunological features of patients with fissured tongue syndrome. $\mathrm{Br} \mathrm{J}$ Oral Maxillofac Surg 1987;25(6):481-7.

4. Kovac Kavic M, Skaleric U. The prevalence of oral mucosal lesions in a population in Ljubljana, Slovenia. J Oral Pathol Med 2000;9:331-5.

5. Richardson, ER. Incidence of geographic tongue and median rhomboid glossitis in 3,319 Negro college students. Oral Surg Oral Med Oral Pathol 1968;26(5):623-5.

6. Vörös-Balog T, Vincze N, Banoczy J. Prevalence of tongue lesions in Hungarian children. Oral Dis 2003;9(2):84-7.

$7 *$. Redman RS. Prevalence of geographic tongue, fissured tongue, median rhomboid glossitis, and hairy tongue among 3,611 Minnesota schoolchildren. Oral Surg Oral Med Oral Pathol 1970;30(3):390-5.

De interés histórico, tiene la peculiaridad de ser el único trabajo encontrado en el que la glositis rómbica media fue diagnosticada en niños, lo que estaría en contra de desechar la hipótesis de una alteración del desarrollo.

8. Kullaa-Mikkonen A, Mikkonen M, Kotilainen R. Prevalence of different morphologic forms of the human tongue in young Finns. Oral Surg Oral Med Oral Pathol 1982;53(2):152-6.

9. Banoczy J, Rigo O, Albrecht M. Prevalence study of tongue lesions in a Hungarian popu- lation. Community Dent Oral Epidemiol 1993;21(4):224-6.

10. Darwazeh AM, Pillai K. Prevalence of tongue lesions in 1013 Jordanian dental outpatients. Community Dent Oral Epidemiol 1993;21(5): 323-4.

11. Sawyer DR, Taiwo EO, Mosadomi A. Oral anomalies in Nigerian children. Community Dent Oral Epidemiol 1984;12(4):269-73.

12. Salem G, Holm SA, Fattah R, Basset S, Nasser C. Developmental oral anomalies among schoolchildren in Gizan region, Saudi Arabia. Community Dent Oral Epidemiol 1987;15(3):150-1.

13. Sedano HO, Carreño Freyre I, Garza de la Garza ML y cols. Clinical orodental abnormalities in Mexican children. Oral Surg Oral Med Oral Pathol 1989;68(3):300-11.

14. Guggenheimer J, Moore PA, Rossie K y cols. Insulin-dependent diabetes mellitus and oral soft tissue pathologies. I. Prevalence and characteristics of non-candidal lesions. Oral Surg Oral Med Oral Pathol Oral Radiol Endod 2000;89(5):563-9.

15*. Kleinman DV, Swango PA, Pindborg JJ. Epidemiology of oral mucosal lesions in United States schoolchildren: 1986-87. Community Dent Oral Epidemiol 1994;22(4):243-53. Interesante estudio en el que se halló que la lengua geográfica era la cuarta lesión oral más frecuente en una población infantil.

16. Arendorf TM, van der Ross R. Oral soft tissue lesions in a black pre-school South African population. Community Dent Oral Epidemiol 1996;24(4):296-7.

17. Garcia-Pola MJ, Garcia-Martin JM, GonzalezGarcia M. Prevalence of oral lesions in the 6year-old pediatric population of Oviedo (Spain). Med Oral 2002;7(3):184-91.

18. Reichart PA. Oral mucosal lesions in a repre- sentative cross-sectional study of aging Germans. Community Dent Oral Epidemiol 2000;28(5):390-8.

19. Jainkittivong A, Aneksuk V, Langlais RP. Oral mucosal conditions in elderly dental patients. Oral Dis 2002;8(4):218-23.

20. Martinez-Diaz AI, Garcia-Pola MJ. Epidemiological study of oral mucosal pathology in patients of the Oviedo School of Stomatology. Med Oral 2002;7(1):4-16.

21. Campisi G, Margiotta V. Oral mucosal lesions and risk habits among men in an Italian study population. J Oral Pathol Med 2001;30(1):22-8.

22. Ikeda N, Handa Y, Khim SP y cols. Prevalence study of oral mucosal lesions in a selected Cambodian population. Community Dent Oral Epidemiol 1995;23(1):49-54.

23. Zain RB, Ikeda N, Razak IA y cols. A national epidemiological survey of oral mucosal lesions in Malaysia. Community Dent Oral Epidemiol 1997;25(5):377-83.

24. Knapp MJ. Oral disease in $\mathbf{1 8 1 . 3 3 8}$ consecutive oral examinations. J Am Dent Assoc 1971;83:1288-93.

25. Bouquot JE. Common oral lesions found during a mass screening examination. $\mathrm{J}$ Am Dent Assoc 1986;112:50-7.

26**. García-Pola MJ, Martínez-Díaz AI. Prevalencia de la patología oral en pacientes de la Escuela de Estomatología de Oviedo. Rev Act Odonto-estomat Esp 1996;56(1):22-31.

Es un estudio de patología oral que incide especialmente en la patología lingual, habiéndose realizado como éste a partir de pacientes que acudieron a una Facultad de Odontología.

27. Weir JC, Davenport WD, Skinner RL. A diagnostic and epidemiologic survey of $\mathbf{1 5 . 7 8 3}$ oral lesions. J Am Dent Assoc 1987;115:439-

RCOE, 2004, Vol 9, №4, 381-390 